\title{
Revision strategies for lumbar pseudarthrosis
}

\author{
Stephen L. Ondra, M.D., AND Shaden Marzouk, M.D. \\ Department of Neurosurgery, Northwestern University, Chicago, Illinois; and Department of \\ Neurosurgery, Henry Ford Hospital, Detroit, Michigan
}

\begin{abstract}
Management and avoidance of lumbar pseudarthrosis are among the most common and challenging tasks faced by reconstructive spine surgeons. The risks of peudarthrosis can be broadly divided into two categories: those within a surgeon's control and those not within his/her control. These include biological factors, graft choices, site preparation, and surgical design. The authors review the biological factors that affect fusion and how they can be manipulated to avoid or manage lumbar pseudarthrosis. Surgical planning and construct design to prevent or treat pseudarthrosis will also be discussed. Additionally, the importance of restoring sagittal balance will be reviewed.
\end{abstract}

KEY WoRdS • pseudarthrosis • lumbar • fusion • revision

Lumbar pseudarthrosis is one of the most common complications encountered in lumbar spine reconstructive surgery. Its avoidance and management are of the most challenging problems in this field. ${ }^{13}$ The rate of pseudar throsis increases based on several parameters. ${ }^{14}$ These include the number of levels fused ${ }^{11}$ the type of fusion, ${ }^{4,5}$ and medical risk factors for pseudarthrosis (Table 1).,6,12, 17,20,29 Pseudarthrosis may be symptomatic or asymptomatic. ${ }^{13}$ There is a strong positive correlation between clinical success and radiologically proven fusion. ${ }^{11}$

In addition to the risks inherent in reconstruction and fusion, a variety of risks are within the surgeon's control. These include the choice of graft material, ${ }^{21}$ preparation of the host graft surface, graft placement environment, ${ }^{35}$ and construct design.

Management of lumbar pseudarthrosis begins with avoidance. If a pseudarthrosis does occur, careful analysis should be given to its cause before considering revision. In revision surgery the surgeon may fix any technical errors of the first procedure, place new, better graft material in the best possible biological environment for fusion, and correct the biomechanical environment to yield the best chance for success. ${ }^{13}$ A surgeon needs to follow the golden rule of surgical revision: if the first procedure does not succeed, do something different with the next.

In this article, we review the strategies to compensate for the individual biological demands present in each patient in whom fusion has been performed. In addition, graft and site preparation choices for optimal fusion and surgical planning will be discussed. Pseudarthrosis management and avoidance will be examined from several perspectives.

Abbreviations used in this paper: $\mathrm{BMP}=$ bone morphogenetic protein; NSAID = nonsteroidal antiinflammatory drug.

\section{REVISION STRATEGIES FOR PSEUDARTHROSIS}

\section{Biological Factors Affecting Fusion Rates}

Various biological factors affect the rate of fusion. These include potentially definable patient behaviors, ${ }^{6,17,20}$ systemic disease processes affecting bone healing, ${ }^{2,19,24,32}$ and medication needs. ${ }^{12,29}$

The most common (and modifiable) patient-related behavior that affects the rate of fusion is smoking. Smoking can lead to a 33\% decrease in the rate of fusion. This is due to vasoconstriction and the restriction of essential small-vessel growth into the fusion site. Strategies to avoid pseudarthrosis begin with elimination of smoking prior to the original procedure. Some surgeons will refuse to perform elective surgery in patients who smoke. Although this is a sound strategy by which to avoid the potential of pseudarthrosis, it may be unrealistic for people in extreme pain or with instability. These patients will find it more difficult to stop smoking than those less severely afflicted because of the added pain-induced stress. It is recommended that smoking cessation classes be offered. While some surgeons will perform surgery if the patient states that he/she has quit, others require testing for a negative blood nicotine level to ensure smoking cessation. Although both are reasonable, neither guarantees the patient's behavior after surgery. Additionally, if someone already suffers from a symptomatic pseudarthrosis, it may not be possible to manage behavior prior to surgery. ${ }^{6,17,20}$

Our strategy is to not begin surgical intervention in moderately symptomatic and structurally stable patients who smoke. All smokers being considered for surgery are referred to smoking cessation clinics. When surgery is planned in a patient who smokes, we assume that they will smoke postoperatively and plan their surgery accordingly. This includes optimization of the graft material, its environment, and circumferential fusion and instrumentation. 
TABLE 1

Rates of pseudarthrosis stratified by number of fusion levels, type of fusion material, and medical risk factors*

\begin{tabular}{cc}
\hline \hline Risk Factor & Rate $(\%)$ \\
\hline no. of fused levels & \\
1 & 18 \\
2 & 26 \\
type of fusion material & \\
autograft & 0 \\
allograft & 6 \\
BMP & 5.5 \\
medical & \\
diabetes & 9 \\
NSAIDs & 55 \\
steroids & 100 \\
smoking & 33 \\
\hline
\end{tabular}

* $\mathrm{BMP}=$ bone morphogenetic protein; NSAIDs = nonsteroidal antiinflammatory drugs.

Biological modifiers, such as BMP and internal or external electrical bone stimulation, ,16,33,36 are also used to enhance the rate of fusion. We have found that aggressive manipulation of the physiological environment can yield good results even in the most challenging cases.

In addition to smoking, other disease processes can have a profound effect on the initial risk of pseudarthrosis, as well as the chance of successful revision when a nonunion does occur. These include osteoporosis, diabetes, rheumatoid arthritis, and other inflammatory bone or softtissue diseases. ${ }^{2,19,24,32}$ Treatments for these disorders involve steroid therapy, NSAIDs, and other medications that can have similarly deleterious effects on bone union. ${ }^{12,29}$ In each of these cases, strategies like those used for smoking can be used to maximize the fusion environment, preventing or managing surgical pseudarthrosis. In addition to physiological management, in all patients in whom there are extraordinary challenges to bone union, the original or revision surgical procedure should be optimized in biomechanical terms. These techniques will be discussed in a proceeding section.

\section{Graft Choice}

The choice of graft material depends on the underlying biological risk, environment of the graft site, and demand on the construct. The gold standard for spinal fusion graft material remains autologous iliac crest. ${ }^{1}$ Other options include autologous fibula and rib, allograft, osteoconductive agents such as coral, and bone modifiers such as BMP. ${ }^{4,30,34,36,37,39}$ Graft choice can be made in part by examining the graft site and assessing the biological demand and likelihood of fusion. These findings can then be measured against the economic and physiological cost of that particular graft material to the patient.

In accordance with the Wolf law, anterior-column grafts are in a much better fusion environment than those in the posterior lateral transverse process. ${ }^{38}$ Although the goldstandard autograft is desirable in any case, the aforementioned benefit of the anterior column graft may make allograft (either free standing or within a disc/vertebral body replacement cage device) a reasonable option in an initial surgical procedure. ${ }^{37}$ If pseudarthrosis has occurred in the presence of allograft, it is recommended that autograft or BMP be used for the revision, even in the anterior column. ${ }^{27}$

In posterior lateral fusion, the barriers to successful arthrodesis are much greater. ${ }^{9}$ The graft is not under compression, vascularity is not as good, and the graft-host interface is less reliable. In this case, autograft remains the standard for the initial and revision surgery. ${ }^{1}$ Local bone can be successfully harvested if its volume and quality are sufficient. The success rate associated with allograft is too poor for it to be used routinely. In general, iliac crest has been the best source for obtaining sufficient volume of high-quality graft. If iliac crest graft has been used in cases in which pseudarthrosis then occurred, it should be used again but the environment must be refined to improve the chance of bone union. This can be achieved by the addition of internal fixation, placement of an interbody graft, ${ }^{10}$ or biological modifiers such as electrical stimulation $^{16,33}$ or an induction agent such as BMP. ${ }^{4,36}$

\section{Host Site Preparation}

An often underappreciated aspect of pseudarthrosis prevention or treatment is preparation of the host fusion site for graft placement. This preparation is essential to establishing an ideal environment to allow bone fusion to occur. Meticulous attention is given to soft-tissue removal from bone surfaces awaiting grafting. This can decrease fibrous ingrowth into the fusion bed..$^{15}$

Arthrodesis is performed to obtain bleeding bone surfaces for all areas onto which graft will be introduced. Special attention is given to joint surfaces; the articular surfaces are cleaned of cartilage and ligamentous tissue. ${ }^{15}$

In anterior-column graft site preparations, the osseous endplate must have all soft tissue removed. Small amounts of bone-related bleeding should occur without complete disruption of the structural integrity of the endplate. ${ }^{28} \mathrm{In}$ this way, ideal site preparation can be accomplished while maintaining structural integrity to minimize later subsidence. Graft surfaces need to be flat in the anterior column to maximize the graft-host contact. Last, the goal is a twothird endplate contact between the graft and host. Soft-tissue removal should be in line with these guidelines regardless of approach - that is, anterior lumbar interbody fusion, transverse lumbar interbody fusion, of posterior lumbar interbody fusion.

\section{Graft Site Placement}

As discussed, graft site placement is dictated by the mechanical needs of the construct and the desire to maximize the physiological environment for fusion. The mechanical demand of a construct can be profoundly affected by the placement of graft material. ${ }^{7}$ Graft placed in the posterior lateral position will not bear load. As such, it has no real impact on the construct. Structural graft material implanted in the anterior column will carry load. By bearing load, anterior grafts decrease the flexion strain on spinal bone fixators such as pedicle screws. These strains could lead to implant-related loosening, pullout, and ultimately construct failure and pseudarthrosis. Anterior grafts load share with the rest of the construct absorbing up to $80 \%$ of the axial load. This load sharing also decreases the risk of implant pullout (Fig. 1). ${ }^{38}$ 


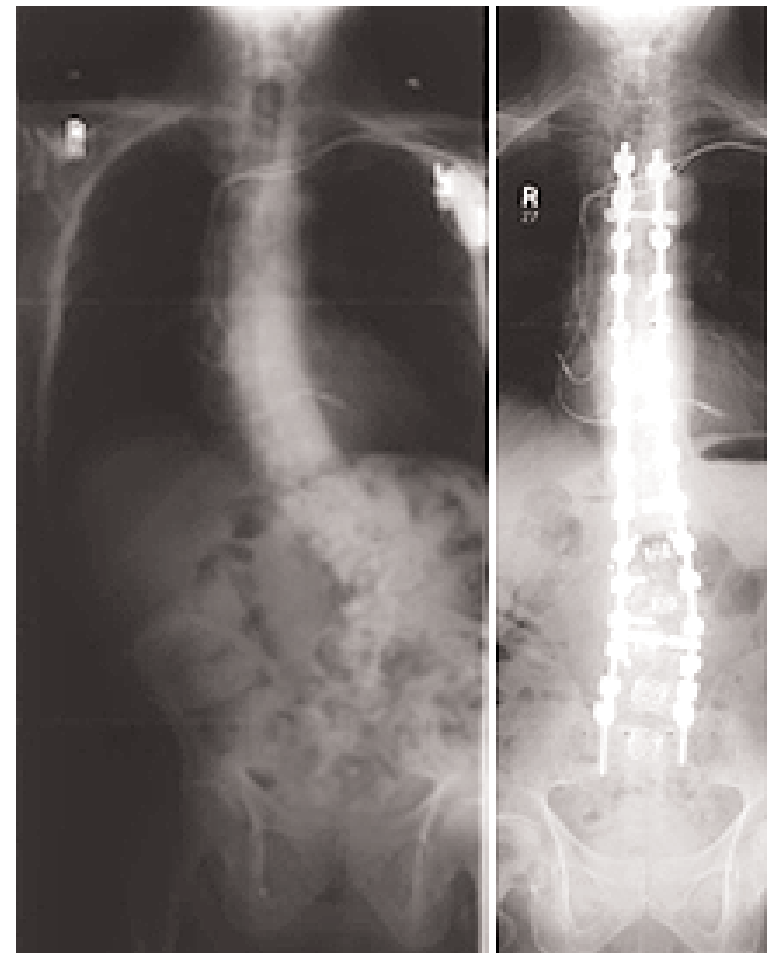

Fig. 1. Left and Right: Radiographic representation demonstrating how load sharing decreases the risk of implant pullout.

The setting can be manipulated by where the graft is placed and how that site was prepared. This can include both posterior lateral grafts and interbody grafts. When pseudarthrosis does occur, attention should be turned to understanding the graft's environment and how it can be improved to allow postrevision healing.

\section{Bone Quality}

Bone quality can deteriorate with systemic diseases such as osteoporosis, malnutrition, hyperparathyroidism, rheumatoid arthritis, and other systemic inflammatory diseases. ${ }^{18,19,24,32}$ It is also affected by medications that inhibit the inflammatory response necessary for healing. ${ }^{12,29}$ These include NSAIDs, steroid medication, and cell-division inhibitors. The degree of bone quality or healing inhibition is one of the foremost factors in the fusion decision process.

Any patient at risk for one of the aforementioned conditions or receiving one of the aforementioned medications needs a dual-energy $x$-ray absorptiometry scan to establish bone mineral density. ${ }^{26}$ Patients with low bone density are at higher risk for pseudarthrosis. Prevention is accomplished by improving the biological and mechanical environments through load sharing the construct with multiple implants, as well as the use of interbody grafting when possible. ${ }^{8}$ In a patient with a symptomatic pseudarthrosis, analysis can establish why bone fusion failed. The response involves improved instrumentation fixation, anterior column load sharing, and biological modifiers to ensure bone fusion.

\section{Surgical Planning and Pseudarthrosis}

One of the most common causes of lumbar pseudarthrosis is poor surgical planning. It is essential that a clear understanding of the global biomechanical abnormalities of the patient's spine be understood prior to surgical planning. In addition to the pathological biomechanics needing correction, surgical decompression and reconstruction create abnormalities. It is important to remember that in most cases instrumentation and fusion take a painful, mobile mechanical abnormality and render it a less painful, nonmobile mechanical abnormality. ${ }^{22}$

In all cases, the goal of surgery is decompression of all symptomatically compressed neurological elements and the creation of a stable balanced spine under normal physiological loads. To achieve this reliably, a detailed surgical plan is created in which the following are assessed: mechanics of the spine, areas of needed decompression, and the effect this will have on the biomechanics in that region. The plan also accounts for the restoration of sagittal and coronal balance. ${ }^{31}$

Sagittal balance is particularly important in preventing lumbar pseudarthrosis as well as in treating it should it develop. ${ }^{25}$ When the spinal plumbline falls in front of the sacrum, the spine is said to be in positive balance (Fig. 2 left). When it falls in back of the sacrum, the balance is negative (Fig. 2 center). When the plumbline falls through the midbody of C-7, L-1, and the sacrum, the spine is said to be in balance (Fig. 2 right). In patients in whom positive sagittal balance is exhibited, an extreme strain is placed on the implants and graft material. As previously discussed, strain decreases the rate of nonunion by decreasing the compressive forces that are ideal for bone fusion. Positive sagittal balance also creates a large cantilever moment arm that places flexion vectors of force on the implants, resulting in failure of the bone-metal inter-
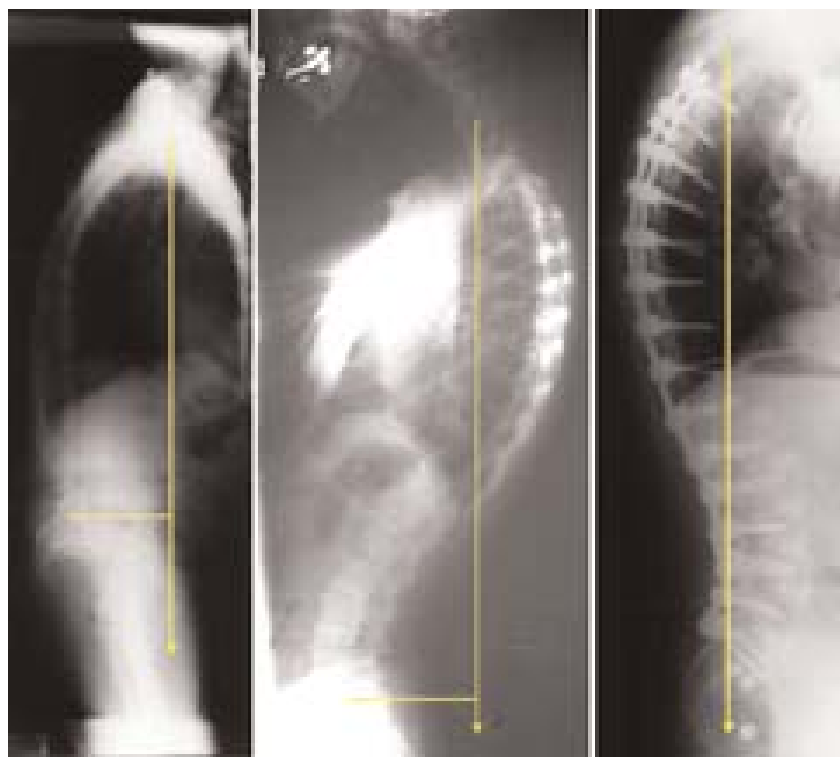

Fig. 2. Lateral radiographs demonstrating positive (left), negative (center), and neutral (right) balance, as indicated by the termination of the plumbline. 
face. This interface failure can lead to construct failure, progression of sagittal imbalance, and pseudarthrosis. ${ }^{25}$

When pseudarthrosis is caused by or results in a positive sagittal-plane deformity, correction is essential. This can be accomplished by lengthening the anterior column, shortening the posterior column, or both. The anterior column can be extended by adding a structural interbody graft to the area of concern (Fig. 3A and B). When combined with a posterior osteotomy and compression the posterior column is shortened. Posterior shortening alone can also be undertaken. The workhorse for nonunion and sagittal-plane correction remains the pedicle subtraction osteotomy. ${ }^{3}$ In this procedure a wedge is removed from the anterior and posterior elements of the spine with the pivot point at the anterior vertebral body line (Fig. 3C and D). This wedge is then closed and sagittal balance is restored. In this procedure the graft is placed under the desired loads for fusion. Consideration of sagittal correction is essential even in routine fusions. It is important to remember that two thirds of the lumbar lordosis occurs between L-4 and S-1. This location is the most common area of treatment for degenerative spinal disease. ${ }^{6}$

In addition to correcting sagittal imbalance, attention must be paid to the principles of host preparation and graft placement for better treatment of pseudarthrosis. When treating a pseudarthrosis and a sagittal-plane disorder, it is important to correct the deformity and, when necessary, extend the fusion. When the fusion extends to the sacrum, iliac fixation should be considered. ${ }^{23}$

The surgical plan also ensures that when possible a fusion does not end until an intact posterior tension band is reestablished. In general, the construct should not end at the level of a laminectomy; nor should it end at a junctional zone. In both cases additional physiological strain is placed on the graft. This can lead to pseudarthrosis and construct failure. This is particularly true for long-segment constructs that create a larger lever arm strain on these junctional zones..$^{10}$ Once a plan is established, it is important to adhere to it to prevent or resolve a pseudarthrosis.

\section{CONCLUSIONS}

The fundamental principle in pseudarthrosis prevention and management involves careful analysis of what could have been done differently, if anything, in the original procedure to prevent the pseudarthrosis. This is combined with a critical assessment of the current biomechanical conditions and requirements to reestablish spinal stability and balance. The surgical plan must allow for correction of all of these factors and the reestablishment of a stable balanced spine. The golden rule of pseudarthrosis repair should be followed: "If something does not work the first time, do something different in revision." This difference consists of improving the environment to allow bone healing. This includes optimal mechanical fixation to prevent graft site motion. The graft must be of an ideal type for the environment into which it is being placed. If the fusion was unidirectional in the original procedure, one can choose contralateral stabilization from the opposite direction. An example of this is a pedicle screw/rod procedure in which nonunion occurs. This can be salvaged by placing an anterior interbody graft with or without posterior rod revision. Conversely, a stand-alone anterior lumbar interbody fusion or posterior lumbar interbody fusion in which fusion fails can be salvaged by undertaking a posterior pedicle screw/fixation and posterolateral fusion. Consideration should always be given to biological modifiers that improve the chance of a successful pseudarthrosis repair. These include electrical stimulation and BMP.

Lumbar pseudarthrosis remains a common and challenging complication in the field of reconstructive spinal surgery. By following fundamental principles, the success rate of repair and stabilization can be maximized.

\section{References}

1. An HS, Lynch K, Toth J: Prospective comparison of autograft vs. allograft for adult posterolateral lumbar spine fusion: differences among freeze-dried, frozen, and mixed grafts. J Spinal Disord 8:131-135, 1995
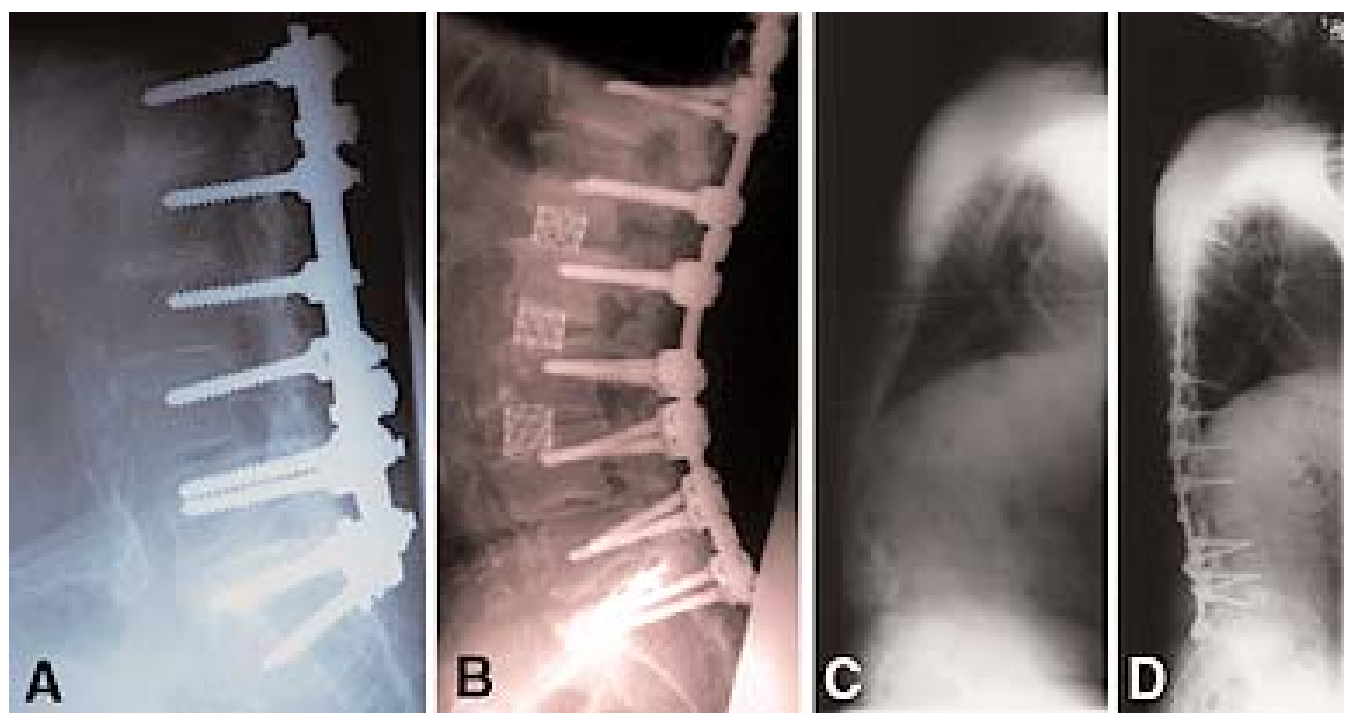

Fig. 3. Lateral radiographs depicting anterior fixation $(A)$, subsequent lengthening of the anterior column by insertion of interbody grafts $(B)$, and posterior shortening of the spine $(C)$ by pedicle subtraction osteotomy $(D)$. 
2. Bendo JA, Spivak J, Moskovich R, et al: Instrumented posterior arthrodesis of the lumbar spine in patients with diabetes mellitus. Am J Orthop 29:617-620, 2000

3. Bridwell KH, Lewis SJ, Lenke LG, et al: Pedicle subtraction osteotomy for the treatment of fixed sagittal imbalance. J Bone Joint Surg Am 85:454-463, 2003

4. Burkus JK, Dorchak JD, Sanders DL: Radiographic assessment of interbody fusion using recombinant human bone morphogenetic protein type 2. Spine 28:372-327, 2003

5. Buttermann GR, Glazer PA, Hu SS, et al: Revision of failed lumbar fusions. A comparison of anterior autograft and allograft. Spine 22:2748-2755, 1997

6. Carpenter CT, Dietz JW, Leung KY, et al: Repair of a pseudarthrosis of the lumbar spine. A functional outcome study. J Bone Joint Surg Am 78:712-720, 1996

7. Cheng CK, Chen CS, Liu CL: Biomechanical analysis of the lumbar spine with anterior interbody fusion on the different locations of the bone grafts. Biomed Mater Eng 12:367-374, 2002

8. Chotivichit A, Fujita T, Wong TH, et al: Role of femoral ring allograft in anterior interbody fusion of the spine. J Orthop Surg 9:1-5, 2001

9. Cripton PA, Jain GM, Wittenberg RH, et al: Load-sharing characteristics of stabilized lumbar spine segments. Spine 25: 170-179, 2000

10. Crock HV: Anterior lumbar interobody fusion: indications for its use and notes on surgical technique. Clin Orthop 165: $157-163,1982$

11. Deguchi M, Rapoff AJ, Zdeblick TA: Posterolateral fusion for isthmic spondylolisthesis in adults: analysis of fusion rate and clinical results. J Spinal Disord 11:459-464, 1998

12. Dimar JR Jr, Ante WA, Zhang YP, et al: The effects of nonsteroidal anti-inflammatory drugs on posterior spinal fusions in the rat. Spine 21:1870-1876, 1996

13. Etminan M, Girardi FP, Khan SN, et al: Revision strategies for lumbar pseudarthrosis. Orthop Clin North Am 33:381-392, 2002

14. Gertzbein SD, Hollopeter MR, Hall S: Pseudarthrosis of the lumbar spine. Outcome after circumferential fusion. Spine 23: 2352-2357, 1998

15. Goldberg VM, Stevenson S: The biology of bone grafts. Semin Arthroplasty 4:58-63, 1993

16. Goodwin CB, Brighton CT, Guyer RD, et al: A double-blind study of capacitively coupled electrical stimulation as an adjunct to lumbar spinal fusions. Spine 24:1349-1357, 1999

17. Hadley MN, Reddy SV: Smoking and the human vertebral column: a review of the impact of cigarette use on vertebral bone metabolism and spinal fusion. Neurosurgery 41:116-124, 1997

18. Heaney RP: The basis for the post-parathyroidectomy increase in bone mass. J Bone Miner Res 17 (Suppl 2):N154-N157, 2002

19. Inaoka M, Tada K, Yonenobu K: Problems of posterior lumbar interbody fusion (PLIF) for the rheumatoid spondylitis of the lumbar spine. Arch Orthop Trauma Surg 122:73-79, 2002

20. Jenkins LT, Jones AL, Harms JJ: Prognostic factors in lumbar spinal fusion. Contemp Orthop 29:173-180, 1994

21. Kaufman HH, Jones E: The principles of bony spinal fusion. Neurosurgery 24:264-270, 1989

22. Konno S, Kikuchi S: Prospective study of surgical treatment of degenerative spondylolisthesis: comparison between decompression alone and decompression with Graf system stabilization. Spine 25:1533-1537, 2000
23. Kuklo TR, Bridwell KH, Lewis SJ, et al: Minimum 2-year analysis of sacropelvic fixation and L5-s1 fusion using S1 and iliac screws. Spine 26:1976-1983, 2001

24. Kumano K, Hirabayashi S, Ogawa Y, et al: Pedicle screws and bone mineral density. Spine 19:1157-1161, 1994

25. Kumar MN, Baklanov A, Chopin D: Correlation between sagittal plane changes and adjacent segment degeneration following lumbar spine fusion. Eur Spine J 10:314-319, 2001

26. Mazess RB, Barden HS: Measurement of bone by dual-photon absorptiometry (DPA) and dual-energy X-ray absorptiometry (DEXA). Ann Chir Gynaecol 77:197-203, 1988

27. Molinari RW, Bridwell KH, Lenke LG, et al: Anterior column support in surgery for high-grade, isthmic spondylolisthesis. Clin Orthop 394:109-120, 2002

28. Polikeit A, Ferguson SJ, Nolte LP, et al: The importance of the endplate for interbody cages in the lumbar spine. Eur Spine J:May online, 2003

29. Sawin PD, Dickman CA, Crawford NR, et al: The effects of dexamethasone on bone fusion in an experimental model of posterolateral lumbar spinal arthrodesis. J Neurosurg (Spine 1) 94:76-81, 2001

30. Sawin PD, Traynelis VC, Menezes AH: A comparative analysis of fusion rates and donor-site morbidity for autogeneic rib and iliac crest bone grafts in posterior cervical fusions. J Neurosurg 88:255-265, 1998

31. Shapiro GS, Taira G, Boachie-Adjei O: Results of surgical treatment of adult idiopathic scoliosis with low back pain and spinal stenosis: a study of long-term clinical radiographic outcomes. Spine 28:358-363, 2003

32. Szpalski M, Gunzburg R: What are the advances for surgical therapy of inflammatory diseases of the spine? Best Pract Res Clin Rheumatol 16:141-154, 2002

33. Tejano NA, Puno R, Ignacio JM: The use of implantable direct current stimulation in multilevel spinal fusion without instrumentation. A prospective clinical and radiographic evaluation with long-term follow-up. Spine 21:1904-1908, 1996

34. Thalgott JS, Klezl Z, Timlin M, et al: Anterior lumbar interbody fusion with processed sea coral (coralline hydroxyapatite) as part of a circumferential fusion. Spine 27:E518-E527, 2002

35. Vardiman AB, Morgan HW, D'Alise MD: Nonunion, in Benzel EC (ed): Spine Surgery: Techniques, Complication Avoidance, and Management. Philadelphia: Churchill Livingstone, 1999, pp 1475-1485

36. Wang JC, Kanim LE, Yoo S, et al: Effect of regional gene therapy with bone morphogenetic protein-2-producing bone marrow cells on spinal fusion in rats. J Bone Joint Surg Am 85: 905-911, 2003

37. Wimmer C, Krismer M, Gluch H, et al: Autogenic versus allogenic bone grafts in anterior lumbar interbody fusion. Clin Orthop 360:122-126, 1999

38. Woodard EJ, Benzel EC: The fundamentals of spine deformity and correction, in Benzel EC (ed): Spine Surgery: Techniques, Complication Avoidance, and Management. Philadelphia: Churchill Livingstone, 1999, pp 1149-1158

39. Yazici M, Asher MA: Freeze-dried allograft for posterior spinal fusion in patients with neuromuscular spinal deformities. Spine 22:1467-1471, 1997

Manuscript received July 16, 2003.

Accepted in final form August 18, 2003.

Address reprint requests to: Stephen L. Ondra, M.D., Department of Neurosurgery, Northwestern University, Suite 20-100, 675 North St. Claire, Chicago, Illinois 60611. email: sondra@nmff.org. 